\title{
The role of dietary protein in optimizing muscle mass, function and health outcomes in older individuals
}

\author{
Robert R. Wolfe* \\ Center for Translational Research in Aging and Longevity, Donald W. Reynolds Institute on Aging, University of Arkansas for \\ Medical Sciences, 4301 West Markham Street, Slot 806, Little Rock, AR 72205, USA
}

(Submitted 31 August 2011 - Final revision received 2 February 2012 - Accepted 17 February 2012)

\begin{abstract}
The determination of whether increased dietary protein can positively affect health outcomes is hindered by the absence of prospective, randomized trials directly addressing this issue in which all pertinent variables are controlled. Consequently, we can only address the question deductively by considering the support for the rationale underlying the notion of a beneficial effect of increased dietary protein intake. With regard to health outcomes, we have focused on older individuals. Muscle mass and function are progressively lost with aging, so that by the age of 60 many individuals have reached a threshold where function begins to be affected. An association between reduced muscle mass and strength and unfavourable health outcomes is more likely to be revealed in individuals who have significant decrements in muscle mass and strength. In this article support for the rationale underlying the notion of a beneficial effect of increased dietary protein intake is considered. Dietary protein intake, and the resulting increased availability of plasma amino acids, stimulates muscle protein synthesis. If all other variables are controlled, increased muscle protein synthesis leads to improved muscle mass, strength and function over time. Increased muscle mass, strength and function are related to improved health outcomes in older individuals. Since adverse effects of reasonable increases in protein intake above the recommended dietary allowance (RDA) of $0 \cdot 8 \mathrm{~g}$ protein $/ \mathrm{kg} / \mathrm{day}$ have not been reported, it is reasonable to conclude that the optimal protein intake for an older individual is greater than the RDA.
\end{abstract}

Key words: Muscle mass: strength: muscle function: protein synthesis

\section{Introduction}

There are few data directly addressing the role of dietary protein in optimizing muscle function and health outcomes in an experimental environment in which all other relevant factors are controlled. Consequently, a systematic approach will be taken to develop a conclusion on this topic that is derived from the current state of knowledge on relevant topics. The first pertinent issue is understanding the metabolic basis by which dietary intake would be expected to influence muscle function. Thus, the role of amino acid availability in regulating the rate of muscle protein synthesis will be discussed. Secondly, evidence will be discussed to substantiate the perspective that stimulated muscle protein synthesis translates to greater mass, strength and function. Finally, the relation between improved muscle mass, strength and function and improved health outcomes will be discussed.

\section{Amino Acid Availability Regulates Muscle Protein Synthesis}

The prevalent thinking through the 1970s and 80s, as expressed in the classic book on protein turnover authored by Waterlow, Garlick and Millward, was that "...control mechanisms for protein synthesis in muscle involve factors other than amino acids"(1). The rationale for this perspective was that low plasma amino acid concentrations may be associated with high or low rates of growth and protein synthesis, and that high amino acid concentrations may be associated with high or low rates of growth and protein synthesis ${ }^{(1)}$. Although the role of amino acids as regulators of muscle protein synthesis is now widely accepted, it is noteworthy that recommendations for amounts of dietary protein have not been significantly revisited since the perspective that amino acids do not regulate protein synthesis was predominant.

Understanding the regulation of muscle protein synthesis and breakdown in human subjects was limited by the available methodologies. We therefore developed a method based on arterial-venous (A-V) sampling and muscle biopsies, utilizing stable isotopes of various naturally-occurring amino acids as tracers, to simultaneously quantify rates of muscle protein synthesis, breakdown, and amino acid trans membrane transport ${ }^{(2)}$. At the same time, we also used the more traditional approach of determining the fractional synthetic rate (FSR) to support results from the A-V sampling approach. 
This series of studies clearly established a predominant role of amino acid availability in the regulation of muscle protein synthesis.

We have shown in a variety of studies that increased availability of amino acids stimulate muscle protein synthesis. This response was evident when amino acids were infused ${ }^{\text {(e.g., 3), }}$ ingested $^{(\mathrm{e} . g ., 4,5)}$, or increased in the context of digested purified protein $\left.{ }^{(\text {e.g., }}{ }^{6}\right)$ or in a beef patty ${ }^{(7)}$. Not only is muscle protein synthesis stimulated acutely by the ingestion of amino acids or protein, chronic supplementation of the diet with amino acids results in an increase in the post-absorptive rate of muscle protein synthesis as well ${ }^{(8)}$. Only the dietary essential amino acids (EAAs) are involved in the stimulation of synthesis. Muscle protein synthesis was stimulated to the same extent when a mixture of EAAs plus nonessential amino acids (NEAAS) in the profile found in beef protein was given in one trial and only the EAA component of the mixture was given in the other trial $^{(9)}$. In contrast, the NEAA component of the mixture failed to elicit any response of muscle protein synthesis ${ }^{(10)}$. The magnitude of response depends on the dose given ${ }^{(3)}$, the profile of the amino acids ${ }^{(11)}$, and the physiological condition of the subject ${ }^{(12)}$. The stimulatory effect of amino acid intake is amplified if combined with the performance of resistance exercise ${ }^{(13)}$. The magnitude of amplification is dependent on the timing of intake in relation to the performance of exercise ${ }^{(14)}$.

The drive of increased amino acid availability to stimulate muscle protein synthesis is powerful enough to overcome, to at least some extent, signals to inhibit synthesis. A striking case in point is the response of children to severe burn injury. Our model to quantify amino acid and protein kinetics in muscle enables the distinction of amino acids appearing intracellularly from protein breakdown as opposed to inward transport ${ }^{(2)}$. Using this model, we have quantified muscle protein and amino acid kinetics in more than 200

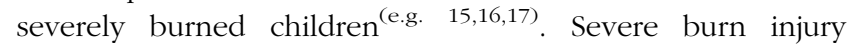
induces a pronounced catabolic state in which there is a net loss of muscle protein that is not readily reversible with nutrition $^{(18)}$. The overall catabolic state and the lack of responsiveness to nutritional intake, the change in the hormonal profile (decreased testosterone, IGF1 and growth hormone) and elevations in cytokines would all suggest that an inhibition of synthesis was a central part of the response. However, studies have consistently shown that muscle protein synthesis is increased following severe burn injury ${ }^{(19)}$. The catabolic state is driven by a large increase in the rate of breakdown. The increased availability of amino acids resulting from accelerated breakdown overcomes, to some extent, the inhibitory signals to synthesis. The stimulation of synthesis is insufficient to overcome the increased rate of breakdown because of the increased release of amino acids to plasma and increased intracellular oxidation. Nonetheless, the increased flux from protein breakdown is sufficient to induce the seemingly paradoxical response of stimulated synthesis in the face of a net catabolic state.

The stimulation of muscle protein synthesis after ingestion or infusion of amino acids is accompanied by an increased flux of amino acids across the cell membrane ${ }^{(20)}$. However, the increased flux of amino acids into the cell and into protein is not necessarily reflected in increased intracellular concentrations. We performed an experiment in which amino acids were infused at three doses into normal volunteers ${ }^{(3)}$. The lowest rate of infusion stimulated muscle protein synthesis, and approximately doubling the rate of infusion resulted in a proportionately greater stimulation of protein synthesis. However, increasing the infusion rate to four times the second rate failed to elicit a further stimulation of synthesis. In response to the two lowest infusion rates intracellular concentrations of free essential amino acids did not increase. It was only in response to the highest infusion rate that the intracellular concentrations increased. Thus, at the lower infusion rates the increased flux of amino acids into the cell was matched by increased flux into protein, so that no change in concentrations resulted. It was only when the inward flux from plasma exceeded the rate of flux into protein (during the highest infusion rate) that intracellular concentrations increased. This study indicates that in the basal state the availability of essential amino acids limits muscle protein synthesis, and when there is increased uptake of amino acids the intracellular concentrations do not increase until protein synthesis is saturated.

It is more difficult to assess the response to decreased availability of amino acids than increased availability. Plasma concentrations of amino acids are generally regulated closely, and even prolonged fasting may not lower levels if the individual has a large enough muscle mass. We used haemodialysis in normal pigs in order to create an experimental paradigm to acutely lower plasma amino acid levels ${ }^{(21)}$. In one group a two hour basal period was followed by four hours of dialysis. The second group also received dialysis for four hours after a basal period of two hours. In addition, amino acids were also infused at a rate adequate to return the plasma concentration to the basal values over the last two hours of dialysis. The rate of muscle protein synthesis closely paralleled the changes in plasma concentrations. Synthesis decreased in response to the decrease in concentration induced by dialysis, and replacement of the lost amino acids during dialysis restored the basal rate of protein synthesis.

Thus, it would seem that there is little doubt that amino acid availability plays a crucial role in regulating muscle protein synthesis. The lack of relationship between both amino acid flux into the cell and flux into protein and the prevailing intracellular amino acid concentrations may explain in part why earlier perceptions about the lack of a role of amino acids has proven to be incorrect.

\section{Stimulated muscle protein synthesis translates to increased mass, strength and function}

Several studies have attempted to address this issue both experimentally and epidemiologically. For example, a recent study indicated that in older individuals increased protein intake corresponds to a slower rate of loss of lean body mass over a three year period ${ }^{(22)}$. However, such studies are conceptually flawed in two respects. In free-living subjects it is difficult to accurately assess dietary patterns, and 
impractical to completely control food intake. Whereas some have nonetheless successfully accomplished complete dietary control for a period of time, accounting for all variables that might affect changes in muscle mass or function in freeliving subjects is virtually impossible. Quantifying the impact of the level of activity is the most challenging problem. Exercise is likely to have at least as much of an effect as the level of protein intake on retention of lean body mass. Accounting for variations in activity level is complicated by the multitude of types of exercise, and the corresponding metabolic consequences. Intense resistance exercise will affect muscle protein differently than aerobic training, and the response to walking will probably differ from both of the other forms of exercise. The timing of protein intake in relation to the performance of exercise will also influence the response ${ }^{(14)}$. Consequently, it is basically impossible to account for self-reported levels of physical activity when evaluating the response to different levels of dietary protein over a prolonged period.

We have performed a series of studies utilizing strict bed rest in order to simulate the consequences of prolonged space flight on skeletal muscle mass and function. In these studies individuals are maintained under complete dietary control and must lie prone for the entire duration of the experiment, which was up to 28 days. Thus, with this experimental protocol we had complete control of activity and dietary intake.

Bed rest induces a drop in basal muscle protein synthesis and breakdown ${ }^{(23)}$. The decrease in breakdown is less than the drop in synthesis, with a loss of muscle mass resulting ${ }^{(23,24)}$. Importantly, the decrease in net protein balance measured acutely with our A-V model across the leg corresponded quantitatively with the amount of muscle loss as estimated by DEXA measurement ${ }^{(24)}$. This provides strong support for the notion that the acute changes in protein synthesis described in the section above correspond to longterm changes in muscle mass, strength and function if all other important variables are maintained constant. To test this hypothesis, normal healthy volunteers were restricted to complete bed rest for 28 days. In both groups energy requirements were estimated by means of the Harris-Benedict equation. This approach enabled maintenance of body weight in both groups throughout bed rest. Carbohydrate, fat and protein intake represented an average of 59, 27 and $14 \%$ of caloric intake from meals. In one group, a dietary supplement containing $16.5 \mathrm{~g}$ EAAs and $30 \mathrm{~g}$ of carbohydrate was given between meals three times a day throughout the 28 days of bed rest. Other dietary intake was modified so that total caloric intake was similar relative to the estimate from the Harris-Benedict equation for both groups. Protein synthesis was measured over an 18 hour duration at the outset of bed rest and after 28 days.

As was predicted from the studies cited above, the EAA-based supplementation increased 18- hour muscle protein synthesis significantly. Further, whereas synthesis dropped on the last day as compared to the first in the control group, in the EAA-supplemented group there was no significant decline in the 18 hour FSR from beginning to end of the bed rest. The increased FSR translated to improvements in all aspects of muscle protein metabolism. Net balance of protein across the muscle was greater in the supplemented group than the controls, and this corresponded to significant differences in leg muscle mass. The differences in leg muscle mass were predicted from the differences in balances over the 18 hour study period. In fact, the EAA supplementation prevented any significant loss in leg muscle mass. Strength (leg extension one repetition maximum) declined in both groups, but less in the group receiving EAA supplementation. Not only was mass and strength significantly improved in the group with the stimulated rates of muscle protein synthesis (i.e., EAA supplementation), but muscle function assessed at the single fiber level was also improved ${ }^{(25)}$.

We utilized the same experimental model to assess the response of elderly people to prolonged (10 days) bed rest $^{(26)}$ and the effect of supplementation with EAAs ${ }^{(27)}$. The overriding goal of this project was to assess the impact of enforced bed rest in older individuals in hospitals or other institutions. The elderly proved to be far more sensitive to the catabolic effects of bed rest than their younger counterparts. Thus, three-times as much muscle was lost in the 10 days of bed rest in the elderly as compared to the 28 day bed rest in the young. This large decrease in muscle mass was consistent with a reduction in muscle protein synthesis of almost $50 \%$. The elderly started the bed rest with much lower functional capacity than the younger subjects, and it was therefore possible to quantify decline in functional status in the elderly. Three standardized tests of function were used to characterize overall functional status (floor transfer, stair ascent, and standing plantar flexion). Decreases in performance of these tests ranged from $20 \%$ (stair ascent) to $80 \%$ (standing plantar flexion) following bed rest. Supplementation of the diet throughout bed rest with the EAA-glucose mixture three times per day, as in the other study, prevented the decrease in muscle protein synthesis that occurs in response to bed rest when extra amino acids or protein are not given. The maintenance of muscle protein synthesis by EAA supplementation prevented the decrements in the performance of any of the functional tests that occurred in the group not receiving supplemental amino acids.

From these studies it is clear that when the most prominent variables with relation to muscle mass and strength (dietary intake and activity level) are completely controlled, stimulated muscle protein synthesis translates to increased muscle mass, strength and function.

\section{Greater muscle mass, strength and function are related to improved health outcomes}

Various boards, such as the Nutrition Board of the National Academy of Sciences (USA) that publish the Dietary Reference Intakes, generally do not recognize differences in requirements for protein intakes for different age groups beyond early childhood. Nonetheless, it is appropriate to focus on the relationships between protein intake, muscle function and health outcomes in the elderly because this group is most vulnerable to deficiencies. Younger individuals may function adequately with a wide range of protein intakes, and have an adequate reservoir of protein to enable an 
adequate response to stress. However, muscle mass and function are progressively lost with aging, so that by the age of 60 many individuals have reached a threshold where function begins to be affected. Substantial loss of muscle mass, strength and function may in turn be associated with impaired health outcomes. Unfavourable health outcomes occur much more frequently in the elderly population than in other age groups. The issue in older individuals is therefore not so much whether the minimal amount of dietary protein needed to avoid clinically-evident deficiencies changes with aging, but rather if beneficial effects in terms of function and health outcomes can be reasonably expected as a result of eating significantly more protein than the recommended dietary allowance (RDA). Since there is little direct experimental evidence on this topic, we will have to rely on developing the rationale supporting the benefits of increased protein intake, particularly in older individuals.

There is a progressive loss of muscle mass with aging. Significant loss of muscle mass with aging is termed sarcopenia. Class I sarcopenia refers to individuals with an amount of lean body mass that is more than one standard deviation below the normal value for a gender-specific young person, and class II sarcopenia refers to the circumstance of lean body mass being more than two standard deviations below the normal value. Whereas sarcopenia is generally considered a syndrome occurring only in elderly, some degree of sarcopenia extends across the age spectrum above 18 years $^{(28)}$. The prevalence of sarcopenia increases progressively until age sixty, where it plateaus at just under $50 \%$ for men and about $60 \%$ for women $^{(28)}$. In a study of over 4,500 individuals 60 years of age and older, Janssen et al. ${ }^{(28)}$ found that the likelihood of functional impairment and disability was approximately two times greater in the older men and three times greater in the older women with Class II sarcopenia than in the older men and women with a normal body mass index (BMI), respectively, even after adjustment for age, race, body mass index, health behaviours, and comorbidity.

The correlation between decreased muscle mass and impaired physical function is not surprising, and several studies support the study cited above. Further, the level of physical function is a major determinant of the quality of life of older individuals. However, whereas most would consider physical function to be an important endpoint, most would not consider physical function itself to be a true health outcome. There are several examples of recent studies that have established a relation between muscle mass or strength and health outcomes. There appears to be an age-dependent threshold in the relationship between muscle mass or strength and health outcomes. The age at which the progression of sarcopenia plateaus corresponds to the age at which muscle loss is sufficient for a threshold effect to become evident in the relation between parameters of muscle mass and strength with health outcomes. For example, Ruiz et al. examined the association between muscular strength and mortality in men in a prospective cohort study of more than 8,000 subjects ${ }^{(29)}$. The average follow up was 18.9 years. They found a strong relationship between strength and all cause mortality as well as mortality from cancer in men over sixty years of age, even after adjustment was made for cardiorespiratory fitness and other potential confounders. In contrast, no such associations were observed in men under 60 years of age ${ }^{(29)}$. The importance of skeletal muscle strength as a predictor of allcause mortality in men is supported by the results of other studies as well ${ }^{(\text {e.g., 30) }}$. The threshold at which muscle loss is sufficient to significantly affect health outcomes may in some circumstances be related to the severity of the disease. Thus, Marquis et al. ${ }^{(31)}$ evaluated mid-thigh muscle cross-sectional area (MTCSA) as a predictor of mortality with chronic obstructive pulmonary disease. They found that MTCSA has a strong impact on mortality in patients with severe disease, but not so much with lesser symptoms.

Evaluation of death risk in over 12,000 haemodialysis patients supports the importance of muscle mass in survival under physiologically stressful circumstances. Serum creatinine arises from muscle, and therefore can be used as an index of muscle mass under normal conditions. However, since poor creatinine clearance is a hallmark of renal disease, one might expect creatinine to be disproportionately higher in renal patients than would be anticipated from their muscle mass. Nonetheless, low (rather than high) creatinine levels are associated with mortality in patients with end stage renal disease ${ }^{(32)}$, thereby strongly supporting the notion that depletion of muscle mass in these patients had a negative impact on outcome. The impact of depletion of lean body mass is further supported by the results of nutritional assessment of approximately 1000 patients admitted to the hospi$\mathrm{tal}^{(33)}$. Low fat-free mass was associated with increased length of stay in the hospital. Further, in a separate study of 101 patients $^{(34)}$, the occurrence of infections in hospitalized elderly patients could be related to the existence of sarcopenia on admission, where a variety of other potential indicators of nutritional status, such as weight, body mass index, albumin level, and C-reactive protein, were ineffective as predictors.

Sarcopenia is conventionally considered in the context of low BMI. As such, it could be argued that it is the low BMI, not specifically the reduced muscle mass, that contributes to impaired health outcomes. However, it has recently become appreciated that a number of older individuals suffer from sarcopenic obesity. In this syndrome, individuals with high BMIs may nonetheless qualify as sarcopenic on the basis of their depleted muscle mass ${ }^{(35)}$. This is true in cancer patients as well as healthy elderly ${ }^{(36)}$. In this setting it was clearly demonstrated by Tan et $a l .{ }^{(36)}$ that depleted muscle mass, per se, is related to reduced survival in patients with cancers of the lung or GI tract. They were able to identify 250 patients with similar BMIs but muscle masses that differed significantly. They showed that sarcopenia was an independent predictor of reduced survival ${ }^{(36)}$. Decreased survival in these patients could have been related to intolerance to chemotherapy, as Prado et al. showed that sarcopenia significantly increased the toxicity of chemotherapy with capecitabine ${ }^{(37)}$.

It is evident from the literature discussed above, as well as a variety of other studies, that there is a strong association between depleted muscle mass and strength and unfavourable health outcomes. It is tempting to conclude from this association that maintenance of muscle mass through 
diet and exercise will reverse those unfavourable outcomes. However, it is also possible that the association between depleted muscle mass and strength and unfavourable health outcomes reflects the catabolic response to stress. Accelerated breakdown of muscle protein is a principle characteristic of cachexia $^{(37)}$. Consequently, it is possible that accelerated muscle protein breakdown occurs in response to severe disease, and the resulting depletion of muscle mass is a reflection of the severity of disease rather than a major contributor to the ultimate outcome. The true situation can only be resolved by prospective, randomized trials in which all variables other than muscle mass and strength are controlled. When all of the potentially confounding variables are considered, such studies would be extremely difficult to perform and interpret.

\section{Summary, Conclusions, and Speculation}

The determination of whether increased dietary protein can positively affect health outcomes is hindered by the absence of prospective, randomized trials directly addressing this issue in which all pertinent variables are controlled. Furthermore, it is hard to imagine that it would ever be possible to perform such a study. We can only address the question deductively by considering the support for the rationale underlying the notion of a beneficial effect of increased dietary protein intake. Increased dietary protein, and the resulting increased availability of plasma amino acids, stimulates muscle protein synthesis. If all other variables are controlled, increased muscle protein synthesis leads to improved muscle mass, strength and function over time. Increased muscle mass, strength and function is related to improved health outcomes in older individuals. Consequently, it is reasonable to deduce that greater protein intake will translate to improved health outcomes, particularly in the elderly. Whereas that cause-effect relationship between muscle mass, strength and function and health outcomes is subject to debate, it is clear that increased muscle mass and function in the elderly translates to an improved ability to perform activities of daily living and therefore quality of life. Improved quality of life is sufficient reason to increase dietary protein intake. Since there is no evidence that a reasonable increase in dietary intake adversely affects health outcomes, and deductive reasoning suggests beneficial effects of a higher protein intake, it is logical to recommend that the optimal dietary protein intake for older individuals is greater than the recommend dietary allowance of $0 \cdot 8 \mathrm{~g}$ protein $/ \mathrm{kg} /$ day.

\section{Financial Disclosure}

Dr. Wolfe has received honoraria from the National Cattleman's Beef Association for giving talks addressing the issue of optimal protein intake levels.

\section{References}

1. Waterlow JC, Garlick PJ \& Millward DJ (1978) Protein Turnover in Mammalian Tissues and in the Whole Body, pp. 666-668. Elsevier/North Holland: Biomedical Press.
2. Biolo G, Gastadelli A, Zhang X-J, et al. (1994) Protein synthesis and breakdown in skin and muscle: a leg model of amino acid kinetics. Am J Physiol Endocrinol Metab 267, E467-E474.

3. Bohe J, Low A, Wolfe RR, et al. (2003) Human muscle protein synthesis is modulated by extracellular but not intracellular amino acid availability: A dose response study. J Physiol 552, 315-324.

4. Volpi E, Ferrando AA, Yeckel CW, et al. (1998) Exogenous amino acids stimulate net muscle protein synthesis in the elderly. J Clin Invest 101, 2000-2007.

5. Volpi E, Mittendorfer B, Wolfe SE, et al. (1999) Oral amino acids stimulate muscle protein anabolism in elderly despite higher first-pass splanchnic extraction. AJP 277, E513-E520.

6. Tipton KD, Elliott TA, Cree MG, et al. (2004) Ingestion of casein and whey proteins result in muscle anabolism after resistance exercise. Med Sci Sports Exerc 36, 2073-2081.

7. Symons TB, Schutzler SE, Coke TL, et al. (2007) Aging does not impair the anabolic response to a protein-rich meal. $\mathrm{AmJ}$ Clin Nutr 86, 451-456.

8. Dillon DL, Sheffield-Moore M, Paddon-Jones D, et al. (2009) Amino acid supplementation increases lean body mass basal muscle protein synthesis and insulin-like growth factor 1 expression in older women. J Clin Endocrinol Metab 94 , $1630-1637$.

9. Volpi E, Kobayashi, Sheffield-Moore M, et al. (2003) Essential amino acids are primarily responsible for the amino acid stimulation of muscle protein anabolism in healthy elderly adults. Am J Clin Nutr 78, 250-258.

10. Katsanos C, Chinkes DL, Paddon-Jones D, et al. (2008) Whey protein ingestion in elderly persons results in greater muscle protein accrual than ingestion of its constituent essential amino acid content. Nutr Res 28, 651-658.

11. Katsansos CS, Kobayashi H, Sheffield-Moore M, et al. (2006) A high proportion of leucine is required for optimal stimulation of the rate of muscle protein synthesis by essential amino acids in the elderly. Am J Physiol Endocriol Metab 291, 381-E387.

12. Tuvdendorj D, Chinkes DL, Zhang X-J, et al. (2011) Skeletal muscle is anabolically unresponsive to an amino Acid infusion in pediatric burn patients 6 months postinjury. Ann Surg 253, 592-597.

13. Biolo G, Maggi SP, Williams D, et al. (1995) Increased rates of muscle protein turnover and amino acid transport after resistance exercise in humans. Am J Physiol Endocrinol Metab 268, E514-E520.

14. Tipton KD, Rasmussen BB, Miller SL, et al. (2001) Timing of amino acid-carbohydrate ingestion alters anabolic response of muscle to resistance exercise. Am J Physiol 281, E197-E206.

15. Herndon DN, Hart DW, Wolf SE, et al. (2001) Reversal of catabolism by beta blockade after burn injury. NEJM $\mathbf{3 4 5}$, 1223-1229.

16. Biolo G, Flemming RYD, Maggi SP, et al. (2002) Inverse regulation of protein turnover and amino acid transport in skeletal muscle of hypercatabolic patients. J Clin Endocrinol Metab 87, 3378-3384.

17. Hart DW, Wolf SE, Ramzy PI, et al. (2001) Anabolic effects of oxandrolone after severe burn. Ann Surg 4, 556-564.

18. Hart DW, Wolf SE, Chinkes DL, et al. (2003) Effect of early excision and aggressive enteral feeding on hypermetabolism catabolism and sepsis after severe burn. J Trauma $\mathbf{5 4}$, 755-761.

19. Wolfe RR (1999) Alterations in protein metabolism due to the stress of injury and infection. In Protein and Amino Acids, pp. 279-284. Washington, DC: National Academy Press. 
20. Miller SL, Chinkes DL, MacLean DA, et al. (2004) In vivo muscle amino acid transport involves two distinct processes. Am J Physiol 287, E136-E141.

21. Kobayashi H, Borsheim E, Traber DL, et al. (2003) Reduced amino acid availability inhibits muscle protein synthesis by a mechanism involving initiation factor eIF2B. Am J Physiol 284, E488-E498.

22. Houston DK, Nicklas BJ, Ding J Harris, et al. (2008) Dietary protein intake is associated with lean mass change in older community-dwelling adults: the Health Aging and Body Composition (Health ABC) Study. Am J Clin Nutr 87, $150 \mathrm{e} 5$.

23. Ferrando A, Lane HW, Stuart CA, et al. (1996) Prolonged bed rest decreases skeletal muscle and whole body protein synthesis. Am J Physiol Endocrinol Metab 270, E627-E633.

24. Paddon-Jones D, Sheffield-Moore M, Urban RJ, et al. (2004) Essential amino acid and carbohydrate supplementation ameliorates muscle protein loss during 28 days bedrest. $J$ Clin Endocrinol Metab 89, 4351-4358.

25. Fitts RH, Ramatowski JG, Peters JR, et al. (2007) The deleterious effects of bed rest on human skeletal muscle fibers are exacerbated by hypercortisolemia and ameliorated by dietary supplementation. Am J Physiol Cell Physiol 293, C313-C320.

26. Kortebein P, Ferrando AA, Lombeida J, et al. (2007) Effect of ten days of bed rest in healthy older men and women. JAMA 297, 1772-1774.

27. Ferrando AA, Paddon-Jones D, Hays NP, et al. (2010) EAA supplementation to increase nitrogen intake improves muscle function during bed rest in the elderly. Clin Nutr 29, $18-23$.

28. Janssen I, Heymsfield SB \& Ross R (2002) Low relative skeletal muscle mass (sarcopenia) in older persons is associated with functional impairment and physical disability. J Am Geriatr Soc 50, 889-896.

29. Ruiz JR, Sui X, Lobelo F, et al. (2008) Association between muscular strength and mortality in men: prospective cohort study. BMJ 337, 439.

30. Metter EJ, Talbot LA, Schrager M, et al. (2002) Skeletal muscle strength as a predictor of all-cause mortality in healthy men. J Gerontol A Biol Med Sci 57, B359-B365.

31. Marquis K, Debigare R, Lacasse Y, et al. (2002) Midthigh muscle cross-sectional area is a better predictor of mortality than body mass index in patients with chronic obstructive pulmonary disease. Am J Respir Crit Care Med 166, 809-813.

32. Lowrie EG \& Lew NL (1990) Death risk in hemodialysis patients: the predictive value of commonly measured variables and an evaluation of death rate differences between facilities. Am J Kidney Dis 15, 458-482.

33. Pichard C, Kyle UG, Morabia A, et al. (2004) Perrier A Vermeulen B Unger P. Nutritional assessment: lean body mass depletion at hospital admission is associated with an increased length of stay. Am J Clin Nutr 79, 613-618.

34. Cosqueric G, Sebag A, Ducolombier C, et al. (2006) Sarcopenia is predictive of nosocomial infection in care of the elderly. Br J Nutr 96, 895-910.

35. Tan BHL, Birdsell LA, Martin L, et al. (2009) Sarcopenia in an overweight or obese patient is an adverse prognostic factor in pancreatic cancer. Clin Cancer Res 15, 6973-6979.

36. Prado CM, Baracos VE, McCargar L, et al. (2009) Sarcopenia as a determinant of chemotherapy toxicity and time to tumor progression in metastatic breast cancer patients receiving capecitabine treatment. Clin Cancer Res 15, 2920-2926.

37. Evans WJ, Morley JE, Argiles C, et al. (2008) Cachexia: A new definition. Clin Nutr 27, 793-799. 